\title{
A Rare Gestational Trophoblastic Disease: Placental Site Trophoblastic Tumor
}

\author{
Senem YAMAN TUNÇ¹, Elif AĞAÇAYAK¹, Mehmet Sait İÇEN¹, Serdar BAŞARANOĞLU², \\ Mehmet Sıddık EVSEN', Yahya AVCI ${ }^{3}$, Zuhat URAKCI ${ }^{4}$, Talip GÜL1 \\ Diyarbakır, Turkey
}

\begin{abstract}
Placental site trophoblastic tumor (PSTT) is a highly rare form of gestational trophoblastic diseases that arise from intermediate trophoblastic cells. By presenting this case, we aimed to review the treatment and diagnosis, approach to PSTT.
\end{abstract}

A 31-year-old (G2P1A1L1) patient had abnormal vaginal bleeding. Serum B-HCG was $5.82 \mathrm{mIU} / \mathrm{ml}$ and the transvaginal USG detected a polypoid mass in uterine cavity. Probe curettage was performed. Histopathologic specimens were confirmed as PSTT. No metastasis was detected. A total abdominal hysterectomy was performed.

PSTT is a rare tumor. In contrast to other trophoblastic tumors, PSTT produces a small amount of $ß$ HCG and it is relatively insensitive to chemotherapy. Adjuvant chemotherapy is suggested to follow surgical treatment in the cases with metastasis.

Keywords: Placental site trophoblastic tumor, Low ß-HCG, Hysterectomy, Chemotherapy

Gynecol Obstet Reprod Med 2016;22(3):171-173 DOI: 10.21613/GORM.2016.485

\section{Introduction}

Placental site trophoblastic tumor (PSTT) is the rarest subtype of gestational trophoblastic neoplasia (GTN), characterized histologically by intermediate cytotrophoblasts (1). It accounts for almost $1-2 \%$ of GTNs with an approximate incidence of $1 / 100.000$ pregnancies. Its mortality rate is $25 \%$ (2). Approximately 200 cases of PSTT have been reported around the world (3). Typically, it occurs in women of reproductive age and may occur after any pregnancy (4). Although a majority of GTNs are extremely sensitive to chemotherapy, PSTT is relatively resistant to it (5). The purpose of this study was to present a 31-year-old case that presented to our clinic with complaints of irregular vaginal bleeding and was diagnosed with PSTT as a result of tests and examinations that were performed.

${ }^{1}$ Dicle University School of Medicine Department of Gynecology and Obstetrics, Diyarbakır

${ }^{2}$ Idil State Hospital Department of Gynecology and Obstetrics, Sirnak

${ }^{3}$ Dicle University School of Medicine Department of Pathology, Diyarbakır

${ }^{4}$ Dicle University School of Medicine Department of Medical Oncology, Diyarbaklr

Address of Correspondence: Senem Yaman Tunç

Dicle University Department of

Gynecology and Obstetrics

Diyarbakir,Turkey

drsenemtunc@hotmail.com

Submitted for Publication:

06. 05.2015

Accepted for Publication:
08. 09. 2015

\section{Case Report}

A 31-year-old case (G2P1A1L1 -Gravida 2, Parity 1, Abortion 1, and Living Children 1) presented to our clinic with irregular vaginal bleeding that was going on for the last 45 days. The patient had a history of spontaneous abortion 15 months ago at about 6 weeks' gestation.

Gynecological examination showed that the patient had an enlarged soft uterus of about 10 weeks' gestation size. Adnexa were found to be normal by bilateral palpation. Speculum examination showed vaginal bleeding in the form of ooze. Serum B-HCG level was $5.82 \mathrm{mIU} / \mathrm{mL}$, and USG scan showed a polypoid structure, irregular in counter and approximately $3 \times 3 \mathrm{~cm}$ in size, extending from the posterior wall of the uterus to the cavity. Bilateral ovaries were normal. There was no sign of ectopic pregnancy in the adnexa. There was no free fluid in the pouch of Douglas, either. Probe-guided curettage was performed in the patient due to abnormal uterine bleeding. Histopathologically, the curettage specimen was interpreted as PSTT.

Thoracic and abdominopelvic CT scan showed no sign of metastasis. Ovary-sparing total abdominal hysterectomy was performed in the patient. Exploration showed that the patient had an enlarged soft uterus of about 10 weeks' gestation size. On the other hand, uterus serosa, bilateral fallopian tubes and ovaries were all normal.

Histopathological examination of the uterine specimen showed a soft yellow-brown lesion with an irregular contour 
extending from posterior wall of the uterus to the cavity. The lesion was $3 \times 3 \times 2 \mathrm{~cm}$ in size and $0,7 \mathrm{~cm}$ away from the uterine serosa (Figure 1). Examination of the endometrial tissue specimen showed tumoral lesions formed by an atypical pleomorphic population of intermediate trophoblastic cells some of which were giant cells penetrating the myometrial muscle fibers and walls of vascular structures. There were several foci of fibrinoid necrosis in the vascular structures (Figure 2). Immunohistochemical examination showed focal positivity for B-HCG and some other areas. Ki-67 proliferation index was $10-15 \%$.

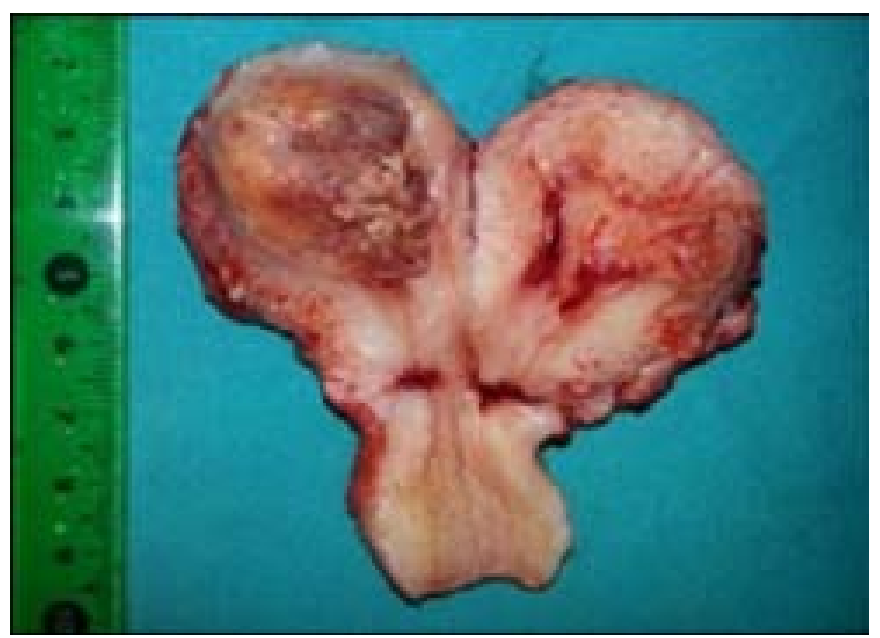

Figure 1: The postoperative photograph of the uterus

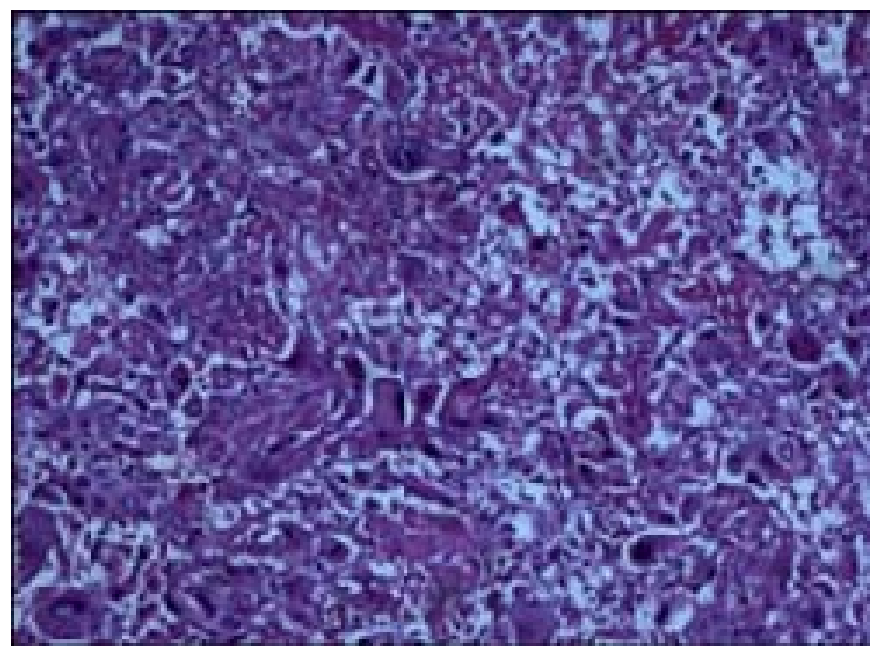

Figure 2: Intermediate trophoblasts with dense eosinophilic cytoplasm and tumor showing extracellular fibrinoid changes (H\&E stain, x200)

The patient, whose postoperative B-HCG level was 1.39 $\mathrm{mlU} / \mathrm{ml}$, was not administered postoperative chemotherapy as the tumor did not reach the uterine serosa and there was no sign of metastasis.

\section{Discussion}

Placental site trophoblastic tumor (PSTT) was first described by Scully and Young in 1981. It was considered to be potentially a malignant neoplasm arising from intermediate trophoblasts (6).

PSTT, which is a very rare subtype of GTN, usually occurs in women of reproductive age. It is rarer in advanced ages. Only 10 postmenopausal cases of PSTT have been reported in the literature. Its pathogenesis is not clearly known; however, it has been reported to follow usually normal term pregnancies and occasionally abortions, GTH, still births and even ectopic pregnancies (7).

The clinical presentation of PSTT is non-specific, which may make the diagnosis difficult. Patients with PSTT usually present with complaints of irregular vaginal bleeding or menorrhagia after an amenorrhea period. On gynecological examination, uterus is usually enlarged in these patients. In addition, serum $\beta$-hCG level is either normal or slightly elevated and is not proportional to the tumor load. Ultrasonography findings are not specific, and final diagnosis requires histological and immunohistochemical studies (8).

It is difficult to predict the clinical course of PSTT. A majority of patients with PSTT do not have metastasis and can be treated simply by curettage. However, at least $15-20 \%$ of patients with PSTT die of widespread metastasis despite intensive multimodal therapy. It mainly metastasizes to the lungs; however, metastasis to the brain, vagina, lymph nodes, liver, kidneys, stomach and spleen has also been reported (9).

Hysterectomy is the standard treatment in patients with PSTT. Preserving the ovaries is important in young women (8). There are several recent studies reporting successful preservation of ovaries in patients with PSTT (10). PSTT has lower sensitivity to chemotherapy compared to other GTNs (11). Postoperative adjuvant chemotherapy may be required in high risk patients. Adverse prognostic factors are as follows: 1- metastasis outside the uterus, 2- at least 2 years of interval between the last pregnancy and the diagnosis of PSTT 3- histological high-grade disease characteristics such as extensive tumor cell necrosis, nuclear atypia, high mitotic count $(>5 / 10$ HPF) and high Ki67 proliferation index, and 4-40 years of age and above (2). The most important one is the metastasis outside the uterus. Prognosis is good in patients with PSTT that is confined to the uterus; however, mortality can be as high as $25 \%$ in patients with PSTT that extends outside the uterus (8). We performed ovary-sparing total hysterectomy in our patient as she was younger than 40 years, and her disease did not extend outside the uterus or demonstrate histological high grade characteristics. The patient received no postoperative chemotherapy, and 1 year follow-up showed no recurrence.

In summary, PSTT is a rare tumor. In contrast to other trophoblastic tumors, PSTT produces a small amount of $\beta-\mathrm{HCG}$ and it is relatively insensitive to chemotherapy $(1,7)$. There is still a lack of consensus in the literature on the key prognostic factors for PSTT, although the time interval since preceding 
pregnancy seems to be the most significant independent predictor of survival, with longer times correlating with worst prognoses (12).

\section{References}

1. Hyman DM, Bakios L, Gualtiere G, Carr C, Grisham RN, Makker V, et al. Placental site trophoblastic tumor: analysis of presentation, treatment, and outcome. Gynecol Oncol 2013;129(1):58-62.

2. Piura B, Shaco-Levy R. Placental site trophoblastic tumor. Harefuah 2007;146(1):62-7.

3. Colecchi C, Partemi S, Minelli N, Cascini F, Rossi R, Fulcheri E, et al. Placental site trophoblastic tumor with lung metastases as cause of death in a young patient: a case report. Placenta 2011;32(12):1060-3.

4. Hassadia A, Gillespien A, Tidy J, Everard RGNJ, Wells M, Coleman R, et al. Placental site trophoblastic tumour: clinical features and management. Gynecol Oncol 2005; 99(3):603-7.

5. Schmid P, Nagai Y, Agarwal R, Hancock B, Savage PM, Sebire NJ, et al. Prognostic markers and long-term outcome of placental-site trophoblastic tumours: a retrospective observational study. Lancet 2009;374(9683):48-55.
6. Scully RE, Young RH. Trophoblastic pseudotumor: a reappraisal. Am J Surg Pathol 1981;5(1):75-6.

7. Ulukuş M, Yılmaz H, Özsaran A, Zekioğlu O, İtil İM, Dikmen Y. Placental site trophoblastic tumor; a case report. J Turk Soc Obstet Gynecol 2008;5(1):40-3.

8. Huang F, Zheng W, Liang Q, Yin T. Diagnosis and treatment of placental site trophoblastic tumour. Int J Clin Exp Pathol 2013;6(7):1448-51.

9. Young RH, Scully R, Mccluskey RA. Distinctive Glomerular Lesion Complicating Placental Site Trophoblastic Tumour: Report of Two Cases. Hum Pathol 1985;16(1):35-42.

10. Liszka L, Wilk M, Wodolazski A, Palen P, Sikora J. Successful treatment of placental site trophoblastic tumor in twin pregnancy without hysterectomy. Tumori 2009;95 (1):108-11.

11. Gupta N, Sharma JB, Mittal S, Talwar D, Kumar L, Kukreja M. Placental Site Trophoblastic Tumor of the Uterus: A Mistaken Diagnosis. JK Science 2010;12(1): $31-2$.

12. Lucas R, Cunha TM. Placental site trophoblastic tumour 3 years after a complete molar pregnancy with atypical localization. J Obstet Gynaecol 2015;35(5):530-2. 